\title{
Stellungnahme der DGAI
}

\section{Rotlichttherapie der allergischen Rhinitis}

\section{Derzeit wird das „Anti-Allergiegerät“ medinose intensiv im Fernsehen beworben. Es soll mittels kurzwelligen Rotlichts zur neben- wirkungsfreien Therapie und auch zur Vorbeugung der allergischen Rhinitis geeignet sein. Was ist an diesen Aussagen wissenschaftlich haltbar?}

$\mathrm{D}$ as „Anti-Allergiegerät" medinose wird vom Unternehmen Medisana (www.medisana.de) vertrieben, vorher war es schon als Bionase (Fa. Buschkuehl) auf dem Markt. Es ist als Medizinprodukt in Apotheken, im Sanitätsfachhandel, aber auch in vielen Kaufhäusern und Supermärkten erhältlich. Es handelt sich um eine Schmalband-Lichtquelle mit vornehmlicher Emission bei $660 \mathrm{~nm}$ Wellenlänge und mit mindestens $4 \mathrm{~mW}$ je Diode Lichtleistung. Die Lichtquelle soll mindestens dreimal täglich für jeweils 4,5 min in jedes $\mathrm{Na}$ senloch eingebracht werden. Laut Hersteller ist das Gerät nicht geeignet für Nasenpolypen. Der Preis liegt bei rund $100 €$. Diese Kosten werden nach Angabe des Herstellers nicht von den gesetzlichen Krankenkassen übernommen.

Das Gerät wurde ursprünglich von der Fa. Amcor in Israel entwickelt. Im Jahr 1997 wurde eine doppelblinde randomisierte prospektive Studie mit dem Gerät veröffentlicht (Neumann I. al. Ann Allergy Asthma Immunol 1997; 78: 399-406). In dieser Studie erhielten 50 Patienten mit perennialer allergischer Rhinitis und zehn Patienten mit Nasenpolypen 14 Tage lang dreimal täglich eine intranasale Belichtung bei $660 \mathrm{~nm}$ mit $4 \mathrm{~mW}$ für 4,4 min (1 J pro Sitzung), während die Kontrollgruppe (29 Patienten mit perennialer allergischer Rhinitis und ein Patient mit Nasenpolypen) lediglich eine Scheinbestrahlung erhielt. Eine Begleitmedikation war nicht erlaubt.

Subjektive und objektive Symptomatik zeigten sich in der Verumgruppe signifikant verbessert. Im Einzelnen er- gaben sich folgende Signifikanzniveaus: nasale Obstruktion 0,016; Kopfschmerzen 0,023; nasaler Juckreiz 0,019; Husten 0,004; Rhinorrhoe 0,0004; allgemeine subjektive Verbesserung 0,021; Nasenschleimhautbefund 0,017; ethmoidales Ödem 0,0007; nasale Passage 0,0002; nasale Sekretion 0,022; allgemeine objektive Verbesserung 0,0006.

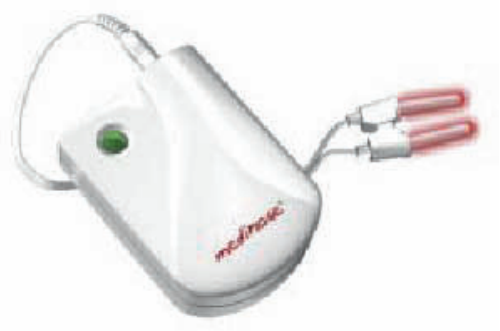

„Anti-Allergiegerät“ medinose: Die beiden Stöpsel werden in die Nasenlöcher platziert, wo sie nach Aktivierung kurzwelliges Rotlicht abstrahlen.

Nach Verumbehandlung zeigte sich bei $72 \%$ der Patienten mit allergischer Rhinitis eine subjektive und bei $70 \%$ eine objektive Verbesserung verglichen mit $24 \%$ bzw. 3\% in der Kontrollgruppe. Die Behandlung war unwirksam bei vorhandenen Nasenpolypen oder $\mathrm{Na}$ senseptumdeviation. Die Autoren folgerten, dass die Schmalband-Rotlichttherapie eine sinnvolle und nebenwirkungsfreie zusätzliche Therapieoption darstellt. Sie postulierten, dass die Wirkung auf der Beeinflussung reaktiver Sauerstoffspezies beruht. Vor einer Rotlichttherapie sollten laut den Autoren der Studie eine Nasenendoskopie und gegegebenenfalls eine Computertomographie durchgeführt werden, um $\mathrm{Na}$ - senpolypen oder eine Septumdeviation auszuschliessen.

Die von den Autoren der Studie vorgeschlagene, aber keineswegs untersuchte, phototherapeutische Wirkungsweise erscheint denkbar (persönliche Mitteilung von Dr. Peter Schröder und Prof. Dr. Jean Krutmann, Institut für umweltmedizinische Forschung der Heinrich-Heine-Universität, Düsseldorf). Dabei käme es während der intranasalen Bestrahlung zu einer vermehrten Produktion von reaktiven Sauerstoffspezies, die wiederum zu einer Verstärkung der antioxidativen Abwehr führt. Dies hätte zur Folge, dass die Auslöseschwelle der allergischen Reaktion erhöht wird und in der Tat eine Verringerung der Symptome allergischer Reaktionen eintreten kann.

Diese Aussagen müssen allerdings sehr kritisch beleuchtet werden. Weder in der Veröffentlichung noch auf der Website des Produkts ist eine Angabe über langfristige Wirkungen, beispielsweise auf Riechvermögen und Immunabwehr, zu finden. Offensichtlich ist auch die notwendige Dosierung nicht geklärt: So wird auf der Produkt-Website die Bestrahlungszeit mit „mindestens dreimal“ oder „zweimal täglich ca. 4,5 Minuten" als nicht verbindlich angegeben.

Fazit: Beim derzeitigen Kenntnisstand kann das Produkt nicht empfohlen werden. Weitere Untersuchungen zu Effektivität, Wirkungsweise und Neben- sowie Langzeitwirkungen sind erforderlich, ehe eine abschließende Beurteilung erfolgen kann.

Priv.-Doz. Dr. Bettina Wedi, Hannover 